\title{
Preparation and Characterization of Novel Drug-Inserted-Montmorillonite Chitosan Carriers for Ocular Drug Delivery
}

\author{
Dongzhi Hou1*, Ruyi Gui', Sheng Hu1, Yi Huang1, Zuyong Feng2* ${ }^{*}$ Qineng Ping ${ }^{3}$ \\ ${ }^{1}$ College of Pharmacy, Guangdong Pharmaceutical University, Guangzhou, China \\ ${ }^{2}$ School of Physics and Optoelectronic Engineering, Guangdong University of Technology, Guangzhou, China \\ ${ }^{3}$ College of Pharmacy, China Pharmaceutical University, Nanjing, China \\ Email: houdongzhi1974@qq.com, ${ }^{*}$ fengzuyong@foxmail.com
}

Received 4 May 2015; accepted 14 June 2015; published 17 June 2015

Copyright (C) 2015 by authors and Scientific Research Publishing Inc.

This work is licensed under the Creative Commons Attribution International License (CC BY).

http://creativecommons.org/licenses/by/4.0/

(c) (i) Open Access

\section{Abstract}

The objective of the study was to investigate the potential of montmorillonite as a sustained carrier in the preparation of drug-loaded nanoparticles for prolonged ocular application. Nanoparticles were prepared by ionic gelation of chitosan with sodium tripolyphosphate (TPP). Betaxolol hydrochloride (BH) was applied as a model drug. TG, FT-IR, TEM, DLS and XRD have been employed in the characterization of drug-encapsulated chitosan-montmorillonite/TPP nanoparticles (CS-MMT/TPP NPs). TEM images showed that the particles were spherical in shape and had a rough surface. The size range of the nanoparticles was between 338 and $585 \mathrm{~nm}$ with positive zeta potential values from $24 \mathrm{mV}$ to $36 \mathrm{mV}$ and encapsulation efficiency values ranging from $12.27 \%$ to $50.92 \%$. In vitro sustained drug release was observed with the BH-loaded nanoparticles in artificial tears (pH 7.4). The results of FT-IR, TG and XRD showed that the drug was coated with CSMMT/TPP NPs. In the mucoadhesion studies, an interaction was found between drug-loaded CSMMT/TPP NPs and mucin, which could enhance precorneal residence time and hence facilitate an effective sustained release. The optimized formulation was determined to be non-irritant and tolerable by modified Draize test. Therefore, the BH-loading CS-MMT/TPP NPs developed are a promising carrier for controlled drug delivery to the eye.

\section{Keywords}

Chitosan, Montmorillonite, Nanoparticles, Mucoadhesive, Sustained Release, Ocular Irritation

\footnotetext{
${ }^{*}$ Corresponding authors.
}

How to cite this paper: Hou, D.Z., Gui, R.Y., Hu, S., Huang, Y., Feng, Z.Y. and Ping, Q.N. (2015) Preparation and Characterization of Novel Drug-Inserted-Montmorillonite Chitosan Carriers for Ocular Drug Delivery. Advances in Nanoparticles, 4, 7084. http://dx.doi.org/10.4236/anp.2015.43009 


\section{Introduction}

It is worth mentioning that primary open-angle glaucoma is the most widespread neuropathy, affecting 60.5 million people in the world [1]. Aside from surgery, the only effective treatment for this disease is IOP-reducing drugs applied topically onto the cornea. The necessity of numerous daily applications of eye-drops for life is serious for patients, besides the risk of side effects in the anterior structures of the eye. Betaxolol hydrochloride $(\mathrm{BH})$, and beta-adrenergic blocking agents, are indicated in the treatment of ocular hypertension and chronic open-angle glaucoma [2]. However, there are a number of challenges in the aspect of the treatment of ocular disease due to certain physiological process which may contribute to the poor efficacy of conventional drug formulations. For instance, blinking and tear drainage through the lachrymal drainage system can act to reduce the residence time of topically applied molecule. Eye-drops placed onto the ocular surface are washed in less than 30 seconds [3]. The corneal epithelium is almost impermeable to any substance larger than 500 Da [4], and most of the commonly used topical drugs are larger than that and consequently cannot cross the cornea. Instead, they permeate throughout the conjunctiva and the underlying sclera in what is known as "non-productive passage”. Altogether, less than 5\% of topically administered drugs reach intraocular tissues [5]. Therefore, developing and designing a novel delivery platform, which suits the requirement of the topical ocular route is necessary.

Chitosan (CS), a cationic polysaccharide polymer, has some important properties such as mucoadhesivity, biocompatibility, nontoxicity and good ocular tolerance [6]. The mucoadhesive character of the polymer makes it increase the drug residence time due to the capacity to adhere to the mucin coat covering the conjunctiva and the corneal surfaces of the eye by non-covalent bonds forming the basis of ocular mucoadhesion [7] [8]. Moreover, CS solutions show pseudoplastic and viscoelastic properties [9] [10], which are desirable for ocular drug administration. It has widely being used in ophthalmic preparations [11]. The report by R. S. Bhatta et al. showed that natamycin-loaded chitosan nanoparticles had the ability to prolong the retention time of drug on the ocular surface [12]. The efficiency of the pharmaceutical treatment of most ocular diseases is limited by short ocular residence of conventional pharmaceutical forms of drugs on the ocular mucosa due to the elimination of drug from the corneal surface by the lachrymal flow. To overcome the problem, ocular administration of drugs in the forms of CS nanoparticles has been proposed. CS-based systems can be used for improving the retention and distribution properties of drugs applied topically in the eye. Thus, all preferable properties make it a unique material for the design of ocular delivery vehicles [13].

Indeed, taking into account the fact that cornea and conjuctiva have a negative charge, it was thought that the use of mucoadhesive polymers which might interact with these extraocular structures would increase the concentration and residence time of the associated drug [14]. The study on cyclosporin A-loaded chitosan nanoparticles has shown the interaction and prolonged residence time of chitosan nanoparticles at the ocular mucosa after the topical administration to rabbits [15], and gatifloxacin-loaded SA-CH nano-reservoir system has also been investigated for the treatment of ocular infections [14].

Montmorillonite (MMT) can form many nanocompsites with different organic compounds. Therefore, it expresses a significant capability to be used as a drug carrier for pharmaceutical purpose [16]. The report by Lin et al. has shown that 5-fluorouracil (5-FU) was successfully intercalated into the interlayer of montmorillonite [17]. In addition, the pillared MMT can be intercalated with ibuprofen as a sustained release carrier [18]. Therefore, MMT material has been proposed as a carrier with high potential for ocular drug delivery.

According to the reports [19] [20], ionic gelation is the most studied and widely used method for fabricating CS nanoparticles, in which cationic chitosan and multivalent polyanions interact to form CS nanoparticles under simple and mild conditions. Among various polyanions, TPP is the most investigated one due to its quick gelling capacity and non-toxicity property. In the present study, the ionic gelation method was used to prepare BHloaded CS-MMT/TPP NPs. Different fabricating conditions were evaluated in terms of their effects on physicochemical properties of BH-loaded CS-MMT/TPP NPs, including morphology, particle size, surface charge, the encapsulation efficiency, loading capacity, as well as release profile. MMT and chitosan were chosen as sustained carriers to achieve better encapsulation efficiency and controlled release profiles of optimized nanoparticles. Therefore, the purpose of this study was to develop a suitable and mucoadhesive system consisting of MMT, chitosan and BH for ocular drug delivery.

\section{Experimental Section}

\subsection{Materials}

Chitosan (deacetylation degree of 95\%) was purchased from HAIDEBEI MARINE BIOEEING CO. LTD, Jinan, 
China. Momtmorillonite was obtained from Zhejiang sanding CO. LTD, china. Betaxolol hydrochloride was purchased from Jinan Haohua Industry, Jinan, China. Male, New Zealand albino rabbits of 2.2 - $2.5 \mathrm{~kg}$ were provided by laboratory animal center, Traditional Chinese medicine university of Guangzhou. All other chemicals were of analytical grade.

\subsection{Acid Activation of Momtmorillonite}

The MMT was activated by treating with $5 \% \mathrm{H}_{2} \mathrm{SO}_{4}$ at the temperature of $40^{\circ} \mathrm{C}$ for $1.5 \mathrm{~h}$. The resulting clays were centrifuged and washed with water several times until they were free of $\mathrm{SO}_{4}^{2-}$, and then were dried at $100^{\circ} \mathrm{C}$ in an air oven until constant weight was attained.

\subsection{Preparation of CS-MMT/TPP NPS}

The nanoparticles were prepared by ionic gelation techniques. In the first step MMT was added to acetum ( $\mathrm{pH}=$ $4.8,2 \% \mathrm{v} / \mathrm{v}$ ) containing betaxolol hydrochloride and heated in water bath at $60^{\circ} \mathrm{C}$ for 6 hours, and then the solution was ultrasonicated for $5 \mathrm{~min}$ and added to chitosan solution and stirred for $1.5 \mathrm{~h}$. subsequently the nanoparticles were fabricated by adding above MMT suspension to chitosan solution $(2 \% \mathrm{v} / \mathrm{v})$ while the TPP solution was added to the mixture suspension with ultrasonication for $10 \mathrm{~s}$ and stirring for 60 mins. The dispersed nanoparticles were frozen at $-80^{\circ} \mathrm{C}$ for $48 \mathrm{~h}$, and then freeze-dried at $-50^{\circ} \mathrm{C}$ for $12 \mathrm{~h}$. Finally, the freeze-dried powders were stored in glass vials for further study.

\subsection{Entrapment Efficiency (EE) and Drug Loading Capacity (DLC)}

To determine the free BH during the preparation of BH-loading CS/TPP NPs and CS-MMT/TPP NPs, the dialyzed solution was collected. The concentration of free BH in the solution was determined by UV-VIS spectrophotometry (UV-1800, MAPADA instrument CO.LTD) using a wavelength of $274.5 \mathrm{~nm}$. The amount of BH in nanoparticles was calculated as the difference between the total amount used to prepare the nanoparticles and the amount present in the supernatant. Fresh prepared BH-loaded CS-MMT/TPP NPs were used for determining the drug-loading capacity. NPs were dissolved in HPLC grade methanol by stirring for $4 \mathrm{~h}$, and the solution was ultrasonicated for 5 mins. This colloidal dispersion was filtered by $0.22 \mu \mathrm{m}$ membrane filter. The drug concentration was analyzed by UV-VIS. The EE and LC of nanoparticles were determined in triplicate and calculated as follows:

$$
\begin{gathered}
\text { Encapsulation Efficiency }(\mathrm{EE} \%)=\frac{(\text { Total amount of BH-Free BH })}{\text { Total amount BH }} \times 100 \% \\
\text { Drug loading capacity }(\text { DLC\% })=\frac{(\text { Total amount of BH-Free BH })}{\text { Total weight NPs }} \times 100 \%
\end{gathered}
$$

\subsection{In Vitro Release Studies}

The release of $\mathrm{BH}$ from nanoparticles was studied by dialysis method in $\mathrm{pH} 7.4$ artificial tears. $2 \mathrm{ml}$ samples were instilled in the dialysis bag which was screwed with two clamps at each end. The dialysis bag was dipped into the receptor compartment containing $35 \mathrm{ml}$ of dissolution medium and stirred continuously at $100 \mathrm{rpm}$ and maintained at $34^{\circ} \mathrm{C}$. The receptor compartment was closed to prevent evaporation of the dissolution medium. Samples were withdrawn at regular time intervals, and the same volume was replaced with fresh dissolution medium. The samples were spectrophotometricially measured at $274.5 \mathrm{~nm}$.

\subsection{Particle Size and Zeta Potential}

NPs size distribution and zeta potential were determined using photon correlation spectroscopy with a Zetasizer 3000 (DelsaNano S, USA). The size distribution analysis was performed at a scattering angle of $90^{\circ}$ at a temperature of $25^{\circ} \mathrm{C}$, and zeta potential was measured by electrophoretic light scattering using a disposable zeta cuvette. All measurements were performed in triplicate and the results were presented as mean $\pm \mathrm{SD}$.

\subsection{Nanoparticles Morphonology}

The morphological examination of nanoparticles was performed on transmission electron microscopy (TEM) 
(JEM-2010HR, Japan). The nanoparticles were applied to a carbon-coated 300 copper grid and then were stained with $2 \%$ phosphotungstic acid for viewing by TEM.

\subsection{Fourier Transform Infrared Spectroscopy (FT-IR)}

FT-IR spectra of freeze-dried nanoparticles were obtained with Bruker Vertex-70 spectrophotometer using potassium bromide $(\mathrm{KBr})$ disk technique. FT-IR spectra of BH-loaded CS-MMT/TPP NPs and their pure components were compared for the evaluation of drug-excipient and excipient-excipient chemical interaction.

\subsection{Thermogravimetric Analysis (TG)}

TG analysis for BH, CS, MMT, acid-MMT, physical mixture and BH-loaded CS-MMT/TPP NPs were performed using a Netzsch STA 409PC TG instrument with NETZSCH-TA4_5 software Proteus Analysis (Germany). The apparatus was calibrated with purified indium (99\%). The samples were placed in flat-bottomed aluminum pan and heated at a constant rate of $10^{\circ} \mathrm{C} / \mathrm{min}$ in nitrogen atmosphere in a temperature range of $30^{\circ} \mathrm{C}$ $1000^{\circ} \mathrm{C}$.

\subsection{X-Ray Diffratometry}

X-ray diffraction patterns for BH, CS, acid-MMT, physical mixture, BH-loaded CS-MMT/TPP NPs were obtained using a Bruker D8 Advance X-ray diffractometer equipped with $\mathrm{CuK} \alpha$. The tube was operated at $40 \mathrm{KV}$ and $40 \mathrm{~mA}$.

\subsection{Mucoadhesion Studies}

Equal volumes of mucin solution and nanoparticles were vortexed for 1 min and zeta potential of the mixture was measured by photon correlation Spectroscopy with a Zetasizer 3000 (DelsaNano S, USA). In this study, the mucoadhesive properties of nanoparticles were evaluated by measuring the change of zeta potential on interaction with negatively charge mucin [21]. The alteration of zeta potential of nanoparticles indicates the interaction with mucin.

\subsection{Ocular Irritation Studies}

The ocular irritancy and damaging effects of NPs and BH were evaluated according to a modified Draize test [22]. Test substance (50 ul) was instilled directly to the cornea in the right eye every $12 \mathrm{~h}$ for 7 days (14 treatments), while left eye severed as control and was treated with normal saline. Observation was carried out before the treatment for every day and was done to evaluate ocular tissue after the last treatment of 1, 2, 4, 24, 48 and $72 \mathrm{~h}$. The congestion, swelling, discharge and redness of the conjunctiva were grade on a scale from 0 to 3,0 to 4,0 to 3 , and 0 to 3, respectively. Irritation and cornea opacity were grade on a scale from 0 to 4 .

\subsection{Statistical Analysis}

The average difference of data was compared by one-way analysis of variance or independent sample t-test (origin 8.5 USA). All data are presented as a mean value with its standard deviation indicated (mean \pm SD). P-values less than 0.05 were considered to be statistical significance.

\section{Result and Discussion}

\subsection{Entrapment Efficiency (EE) and Drug Loading Capacity (DLC)}

The effect of CS concentration on the encapsulation efficiency and drug-loading capacity were examined as shown in Table 1. The increasing concentration of CS from $1.0 \mathrm{mg} / \mathrm{ml}$ to $1.5 \mathrm{mg} / \mathrm{ml}$ at a constant TPP concentration and chitosan to TPP mass ratio, caused a great increase in drug content from $30.32 \%$ to $39.51 \%$. This indicates that a less amount of nanoparticles were formed due to the lower chitosan concentration. However, when the concentration of CS was increased from $1.5 \mathrm{mg} / \mathrm{ml}$ to $3.0 \mathrm{mg} / \mathrm{ml}$, a significant decrease in the encapsulation efficiency from $39.51 \%$ to $31.6 \%$ was exhibited. This may be explained by the fact that relatively higher viscosity of the higher CS concentration hinders the drug encapsulation [23]. With the similar results, the loading 
Table 1. Effect of chitosan concentration on the encapsulation efficiency and drug loading capacity for the prepared nanoparticles (mean $\pm \mathrm{SD}$ ).

\begin{tabular}{cccc}
\hline Formula code & CS concentration $(\mathrm{mg} / \mathrm{ml})$ & Encapsulation efficiency (\%) & Loading capacity (\%) \\
\hline 1 & 1 & $30.32 \pm 0.12$ & $11.49 \pm 0.33$ \\
2 & 1.5 & $39.51 \pm 0.23$ & $8.67 \pm 0.45$ \\
3 & 2 & $33.32 \pm 0.13$ & $6.68 \pm 0.78$ \\
4 & 2.5 & $33.01 \pm 0.21$ & $5.99 \pm 0.13$ \\
\hline
\end{tabular}

Particles preparation conditions: the weight of montmorillonite $=5 \mathrm{mg}, \mathrm{BH}$ concentration $=1.25 \mathrm{mg} / \mathrm{ml}, \mathrm{chitosan} / \mathrm{TPP}=10: 3$, $\mathrm{pH}=4.8$.

capacity firstly increased with the increase of the CS concentration and then decreased. The reason appears to be similar to the above definition. In addition, the increase of the weight of CS and the corresponding change of encapsulation efficiency were a contributor to the result as well. The effects of chitosan to TPP mass ratio on the drug content and loading capacity of nanoparticles were studied at the mass ratio of 10:1, 10:1.5, 10:2, 10:2.5 and 10:3 at a fixed TPP concentration of $1.0 \mathrm{mg} / \mathrm{ml}$, and the results were presented in Table 2. The encapsulation efficiency and loading capacity of nanoparticles decreased with the decrease in weight ratio of CS/TPP from 10:1 to 10:2. A possible explanation may be that a more compact solid-matrix structure due to the increased mass ratio for CS/TPP, which leads to the increasing amount of formed nanoparticles, resulting in the increase for encapsulation efficiency and loading capacity in nanoparticles. However, when the mass ratio is over the critical point (10:2), the aggregation of nanoparticles occurs, which indicated the reduction of encapsulation efficiency. The influence of the different amount of MMT on drug content and drug loading capacity was also examined, as shown in Table 3. Table 3 shows that encapsulation efficiency increased from 5.5\% to $9.21 \%$, when initial weight of MMT increased from $1 \mathrm{mg}$ to $5 \mathrm{mg}$. However, the further increase of MMT amount does not lead to a sequential increase of encapsulation efficiency. The reason may be the more drugs adsorbed and intercalated with the higher MMT weight could be a contributor to the increase. When the amount of MMT was increased to some value, the drug content appears to keep a constant value possibly due to the redundant MMT which does not participate in the formation of the nanoparticles. The effect of initial $\mathrm{BH}$ concentration on drug content and loading capacity of the nanoparticles were also examined, as shown in Table 4. The increase of BH concentration was done from $0.75 \mathrm{mg} / \mathrm{ml}$ to $1.25 \mathrm{mg} / \mathrm{ml}$, accompanied with the increasing encapsulation from $3.12 \%$ to $12.44 \%$ (Table 4). However, when BH concentration reaches $1.25 \mathrm{mg} / \mathrm{ml}$, the drug content tends to decrease with progressive enhancement of drug loading capacity. This indicates that BH adsorption and encapsulation by electrostatic interactions normally exhibit a saturation kinetics which reaches a peak value at high concentration under constant temperature. On the basis of single-factor test, orthogonal test was carried out by choosing four factors and each factor had three levels (Table 5). Preparation technology was optimized through the orthogonal experiment. According to the analysis of variance, the influence factors of drug content of the nanoparticles were followed by the sequence: TPP concentration $>$ chitosan concentration $>$ drug concentration $>$ chitosan to TPP mass ratio. The optimized preparation was chosen for further study.

\subsection{Effect of Chitosan Concentration on In-Vitro Release Performance}

Figure 1 shows $\mathrm{BH}$ release profiles in the respect of different chitosan concentration during $\mathrm{BH}$ encapsulation. The reduction of initial burst release with the chitosan concentration increasing from $1.0 \mathrm{mg} / \mathrm{ml} \mathrm{to} 2.0 \mathrm{mg} / \mathrm{ml}$ during $\mathrm{BH}$ encapsulation was observed. This may be explained by the fact that the increased viscosity at higher chitosan concentration allowed the formation of denser nanoparticles upon the interaction with TPP, resulting in greater crosslink density and less swelling ability, and consequently reducing the BH release [24]. In addition, the decrease of encapsulation efficiency with the increase of chitosan concentration may be a contributor to this phenomenon. With the durative increase chitosan concentration, the increase of initial burst release for nanoparticles was observed due to the aggregation of nanoparticles caused by the increased chitosan concentration resulting in the more loose structure for nanoparticles.

\subsection{Effect of Amount Varies of Montmorillonite on In-Vitro Release Performance}

The effect of different amount of montmorillonite on BH release from prepared nanoparticles was studied. From 
Table 2. Effect of chitosan to TPP mass ratio on the encapsulation efficiency and drug loading capacity for the prepared nanoparticles (mean $\pm \mathrm{SD}$ ).

\begin{tabular}{cccc}
\hline Formula code & Mass ratio of CS to TPP & Encapsulation efficiency (\%) & Loading capacity (\%) \\
\hline 1 & $10: 1$ & $45.63 \pm 0.11$ & $13.87 \pm 0.78$ \\
2 & $20: 3$ & $43.77 \pm 0.36$ & $12.64 \pm 0.34$ \\
3 & $10: 2$ & $39 . .82 \pm 0.23$ & $11.58 \pm 0.46$ \\
4 & $20: 5$ & $39.41 \pm 0.21$ & $11.49 \pm 1.26$ \\
5 & $10: 3$ & $36.51 \pm 0.15$ & $11.15 \pm 0.27$ \\
\hline
\end{tabular}

Particles preparation conditions: the weight of montmorillonite $=5 \mathrm{mg}, \mathrm{BH}$ concentration $=1.25 \mathrm{mg} / \mathrm{ml}$, chitosan concentration $=2 \mathrm{mg} / \mathrm{ml}, \mathrm{pH}=4.8$.

Table 3. Effect of the amount of MMT on the encapsulation efficiency and drug loading capacity for the prepared nanoparticles (mean $\pm \mathrm{SD}$ ).

\begin{tabular}{cccc}
\hline Formula code & MMT (mg) & Encapsulation efficiency (\%) & Loading capacity (\%) \\
\hline 1 & 1 & $19.29 \pm 0.25$ & $5.5 \pm 0.21$ \\
2 & 3 & $26.96 \pm 0.32$ & $7.55 \pm 0.34$ \\
3 & 5 & $35.47 \pm 0.12$ & $9.21 \pm 0.46$ \\
4 & 7 & $37.13 \pm 0.45$ & $9.38 \pm 0.67$ \\
5 & 10 & $37.75 \pm 0.79$ & $9.48 \pm 0.43$ \\
\hline
\end{tabular}

Particles preparation condition: chitosan concentration $=2 \mathrm{mg} / \mathrm{ml}, \mathrm{BH}$ concentration $=1.25 \mathrm{mg} / \mathrm{ml}$, chitosan $/ \mathrm{TPP}=10: 3$, $\mathrm{pH}=4.8$.

Table 4. Effect of various initial drug concentration on the encapsulation efficiency and drug loading capacity for prepared nanoparticles (mean $\pm \mathrm{SD}$ ).

\begin{tabular}{cccc}
\hline Formula code & Drug concentration $(\mathrm{mg} / \mathrm{ml})$ & Encapsulation efficiency (\%) & Loading capacity (\%) \\
1 & 0.75 & $11.92 \% \pm 0.13 \%$ & $5.12 \% \pm 0.12 \%$ \\
2 & 1.0 & $17.20 \% \pm 0.21 \%$ & $12.44 \% \pm 0.01 \%$ \\
3 & 1.25 & $34.72 \% \pm 0.11 \%$ & $10.20 \% \pm 0.77 \%$ \\
4 & 1.5 & $23.79 \% \pm 0.26 \%$ & $10.09 \% \pm 0.39 \%$ \\
\hline
\end{tabular}

Particles preparation conditions: the weight of montmorillonite $=5 \mathrm{mg}$, chitosan concentration $=1.25 \mathrm{mg} / \mathrm{ml}, \mathrm{chitosan}: \mathrm{TPP}=10: 3 . \mathrm{pH}=4.8$.

Table 5. Effect of chitosan concentration, TPP concentration, mass ratio of chitosan/TPP and BH concentration on the encapsulation efficiency, loading capacity, particle size, zeta potential values and PID (mean \pm SD).

\begin{tabular}{ccccccccccc}
\hline code & $\mathrm{A}^{\mathrm{a}}$ & $\mathrm{B}^{\mathrm{b}}$ & $\mathrm{C}^{\mathrm{c}}$ & $\mathrm{D}^{\mathrm{d}}$ & $\mathrm{EE}(\%)$ & $\mathrm{LC}(\%)$ & $\begin{array}{c}\text { Particle size } \\
(\mathrm{nm})\end{array}$ & $\begin{array}{c}\text { Zeta Potential } \\
(\mathrm{mV})\end{array}$ \\
\hline 1 & 1.0 & 0.8 & $10: 1$ & 0.75 & $13.10 \pm 0.21$ & $5.26 \pm 0.16$ & $338.4 \pm 2.3$ & $25.14 \pm 3.6$ & $0.254 \pm 0.11$ \\
2 & 1.0 & 1.0 & $10: 1.5$ & 1.25 & $15.17 \pm 0.11$ & $8.20 \pm 0.34$ & $366.5 \pm 5.6$ & $30.31 \pm 2.7$ & $0.213 \pm 0.08$ \\
3 & 1.0 & 1.2 & $10: 2.5$ & 2 & $18.00 \pm 0.32$ & $11.49 \pm 0.31$ & $585.5 \pm 7.8$ & $24.62 \pm 1.2$ & $0.282 \pm 0.23$ \\
4 & 1.5 & 0.8 & $10: 1.5$ & 2 & $19.53 \pm 0.23$ & $10.56 \pm 0.24$ & $355.8 \pm 4.3$ & $29.49 \pm 4.8$ & $0.22 \pm \pm 0.34$ \\
5 & 1.5 & 1.0 & $10: 2.5$ & 0.75 & $12.27 \pm 0.13$ & $3.49 \pm 0.38$ & $529.3 \pm 2.2$ & $31.82 \pm 2.1$ & $0.260 \pm 0.21$ \\
6 & 1.5 & 1.2 & $10: 1$ & 1.25 & $35.31 \pm 0.42$ & $14.85 \pm 0.56$ & $480.5 \pm 4.5$ & $30.70 \pm 3.9$ & $0.282 \pm 0.13$ \\
7 & 2.0 & 0.8 & $10: 2.5$ & 1.25 & $36.11 \pm 0.35$ & $11.28 \pm 0.43$ & $503.5 \pm 5.9$ & $25.27 \pm 4.2$ & $0.242 \pm 0.22$ \\
8 & 2.0 & 1.0 & $10: 1$ & 2 & $13.40 \pm 0.12$ & $6.33 \pm 0.57$ & $451.7 \pm 6.7$ & $29.40 \pm 5.6$ & $0.220 \pm 0.26$ \\
9 & 2.0 & 1.2 & $10: 1.5$ & 0.75 & $50.92 \pm 0.48$ & $11.59 \pm 0.24$ & $524.3 \pm 8.2$ & $36.42 \pm 2.3$ & $0.275 \pm 0.15$ \\
\hline
\end{tabular}

${ }^{\mathrm{a}}$ Chitosan concentration; ${ }^{\mathrm{b}} \mathrm{TPP}$ concentration; ${ }^{\mathrm{c}} \mathrm{Chitosan} / \mathrm{TPP}$ mass ratio; ${ }^{\mathrm{d}} \mathrm{BH}$ concentration. 


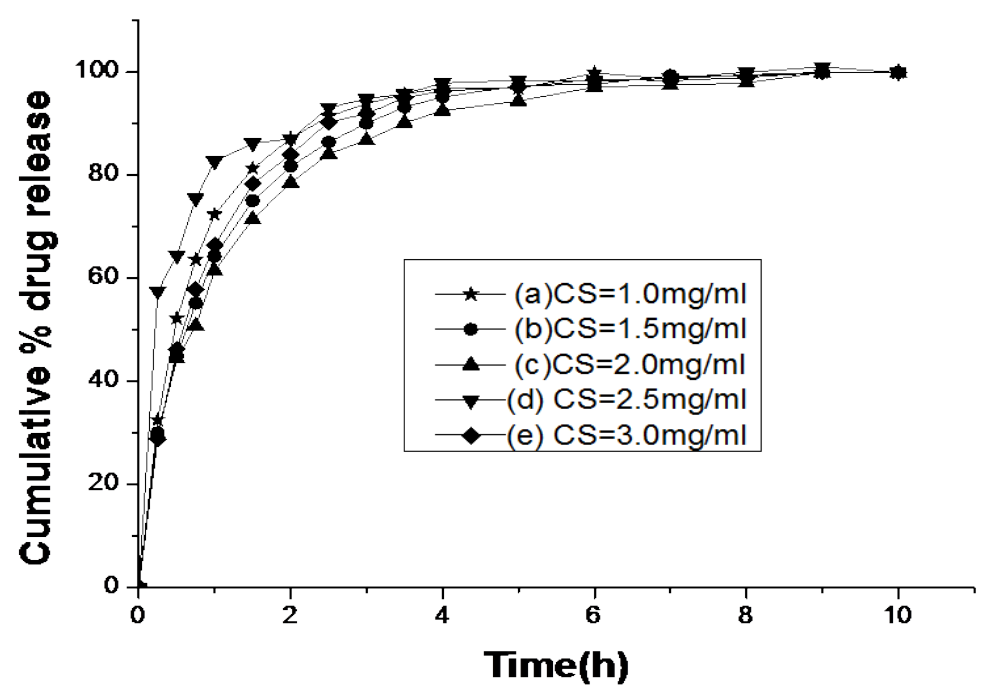

Figure 1. The influence of different chitosan concentration (a) chitosan $=1.0$ $\mathrm{mg} / \mathrm{ml}$; (b) chitosan = $1.5 \mathrm{mg} / \mathrm{ml}$; (c) chitosan = $2.0 \mathrm{mg} / \mathrm{ml}$; (d) chitosan $=2.5$ $\mathrm{mg} / \mathrm{ml}$; (e) chitosan $=3 \mathrm{mg} / \mathrm{ml}$ ) on BH release from prepared nanoparticles.

the release profiles in Figure 2, we can see that the overall rate of drug release (i.e. the release fraction of drug content of nanoparticles at a given time) tended to become lower as the amount of MMT increasing from $1 \mathrm{mg}$ to $5 \mathrm{mg}$, while it started to become higher with the further increase of the weight of MMT. It is obvious that the amount of MMT should be in an appropriate range, which can only effectively form nanoparticles with chitosan and TPP. This indicates that MMT at high amount would interrupter the formation of denser chitosan nanoparticles due to the hydroxyl group in the MMT interacting with the amino of chitosan, which disturbed the electrostatic interaction between chitosan and TPP. These reasons resulted in more fast release of BH from nanoparticles.

The result of drug release experiment for $\mathrm{BH}$ solution and optimized NPs in stimulated ocular circumstances $\left(37^{\circ} \mathrm{C}, \mathrm{pH} 7.4\right)$ are shown in Figure 3. We can observe that BH-loaded CS-MMT/TPP NPs showed a lower burst release as compared to BH-loaded CS/TPP NPs, but both presented a complete release of BH over a period of $10 \mathrm{~h}$. For pure BH solution, which showed a fast release profile with $100 \% \mathrm{BH}$ released within $2 \mathrm{~h}$. Hence, NPs exhibited significantly sustained release in comparison to drug solution. In addition, this could also indicate that CS-MMT/TPP NPs showed better sustained-release compared to that of CS/TPP NPs. From the release profiles of NPs, we may come to a conclusion that BH encapsulated in the matrices of nanoparticles are released by three mechanisms taking place in sequence: 1) BH desorption from the particle surface; 2) diffusion and readsorption of the BH through the pores of the polymer network or the interlayer of montmorillonite; 3) degradation and erosion of the polymeric network.

\subsection{Particle Size and Zeta Potential}

The reduced size of nanoparticle formulation was very interesting in the view of their application in ocular drop formulations. It is well known that the size of particle under $10 \mu \mathrm{m}$ would not cause any ocular irritation. Therefore, the reduction achieved in particles size could result in more compliance for patient. In this study, all the nanosystems ranged in diameter from $338.4 \pm 2.3$ to $585.5 \pm 7.8 \mathrm{~nm}$ with a polydispersity index values between $0.213 \pm 0.08$ to $0.282 \pm 0.13$, as shown in Table 5 .

The effect of concentration and weight ratio of CS and TPP on the mean nanoparticle diameter were examined. The nanoparticle diameter appears to be dependent on the concentration of CS and TPP and chitosan to TPP mass ratio, the minimum diameter $(338.4 \mathrm{~nm})$ corresponding to the lowest CS and TPP concentration, and maximum diameter $(585.5 \mathrm{~nm})$ corresponding to the highest TPP concentration and weight ratio between chitosan and TPP. The increase CS concentration from $1.0 \mathrm{mg} / \mathrm{ml}$ for formula 1 to $1.5 \mathrm{mg} / \mathrm{ml}$ for formula 4 , then 2.0 $\mathrm{mg} / \mathrm{ml}$ for formula 7 at the constant TPP concentration, showed a significant increase in the particle diameter from 338 to 355, then $503 \mathrm{~nm}$, respectively. The increased particle diameters were attributed to the increase of 


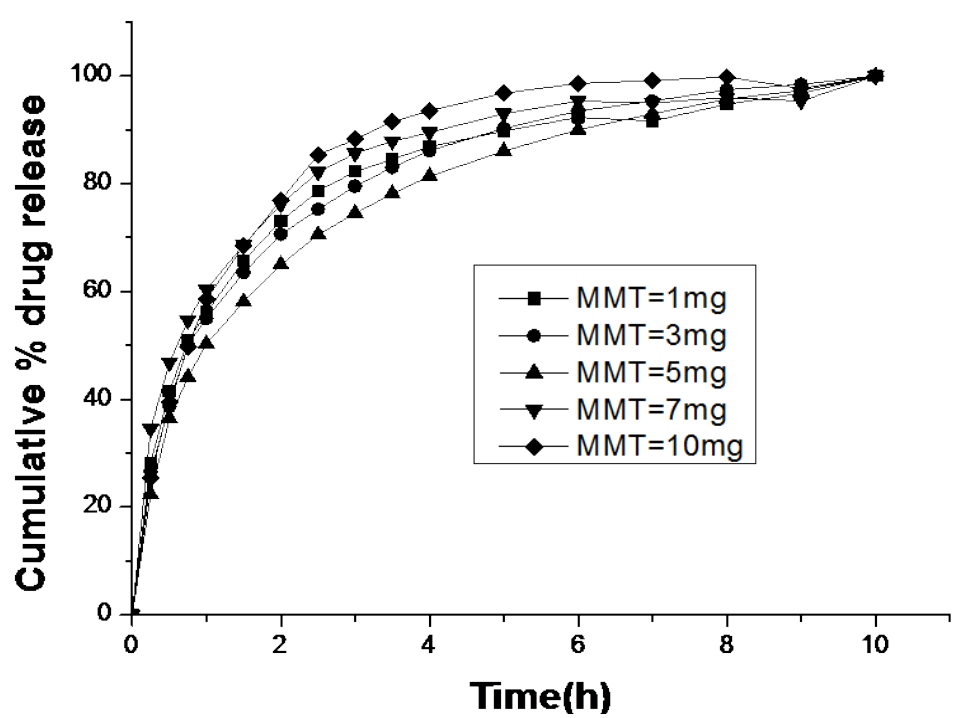

Figure 2. In vitro release profiles of different amount of acid MMT prepared nanoparticles.

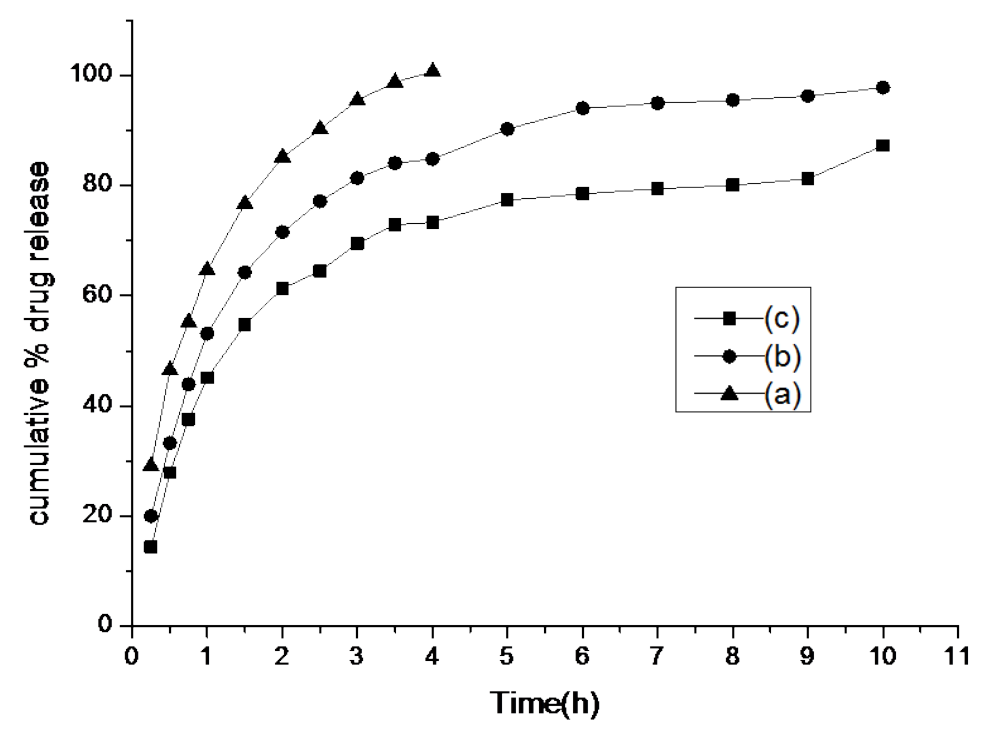

Figure 3. In vitro release profiles of (a) BH, (b) BH loading CS/TPP NPs,(c) BH loading CS-MMT/TPP NPs.

CS viscosity [25] and the higher availability of protonated amine groups for ionic gelation with increasing CS concentration [14].

Zeta potential studies of the nanoparticles showed the values ranging from $24.62 \pm 1.2$ to $36.42 \pm 2.3 \mathrm{mv}$ (Table 5). The positive zeta-potential values indicate that the surface of the nanosystems is mostly composed of CS [26]. This net positive charge of the particle is desirable to promote electrostatic interaction with the ocular mucin [27]. In general, it was noted that zeta potential of the nanoparticles decreased as the CS/TPP ratio decreased. The lower zeta potential with increasing TPP amounts might be caused by an increased masking of free positively changed amino groups of CS. In addition, increasing CS concentration results in an increase of zeta potential of the nanoparticles due to the increase of free positively charged amino groups of CS. According to the analysis of variance (data not shown), the effect factors on nanoparticles size and zeta potential take place in sequence: chitosan to TPP mass ratio $>$ TPP concentration $>$ chitosan concentration $>$ drug concentration and chitosan to TPP mass ratio $>$ chitosan concentration $>$ TPP concentration $>$ drug concentration, respectively. 


\subsection{Nanoparticles Morphology}

A representative TEM image for drug-loaded CS-MMT/TPP nanoparticles was presented in Figure 4. The results indicated that the tested nanoparticles were predominantly spherical in the shape with an irregular surface. The diameter of nanoparticles was about $250 \mathrm{~nm}$ smaller than the diameter determined by photon correlation spectroscopy, in which this could have been expected since the fresh prepared nanoparticles suspensions for the photon correlation spectroscopy experiments, and the chitosan and MMT have the ability to swell in contact with acetic acid, while the TEM experiments were performed with dry samples.

\subsection{Fourier Transforms Infrared Spectroscopy (FT-IR)}

$\mathrm{BH}$, chitosan, montmorillonite, their physical mixture, BH-loaded CS/TPP NPs and BH-loaded CS-MMT/TPP NPs were analyzed using FT-IR spectrophotometer for characteristic absorption bands (Figure 5). The IR

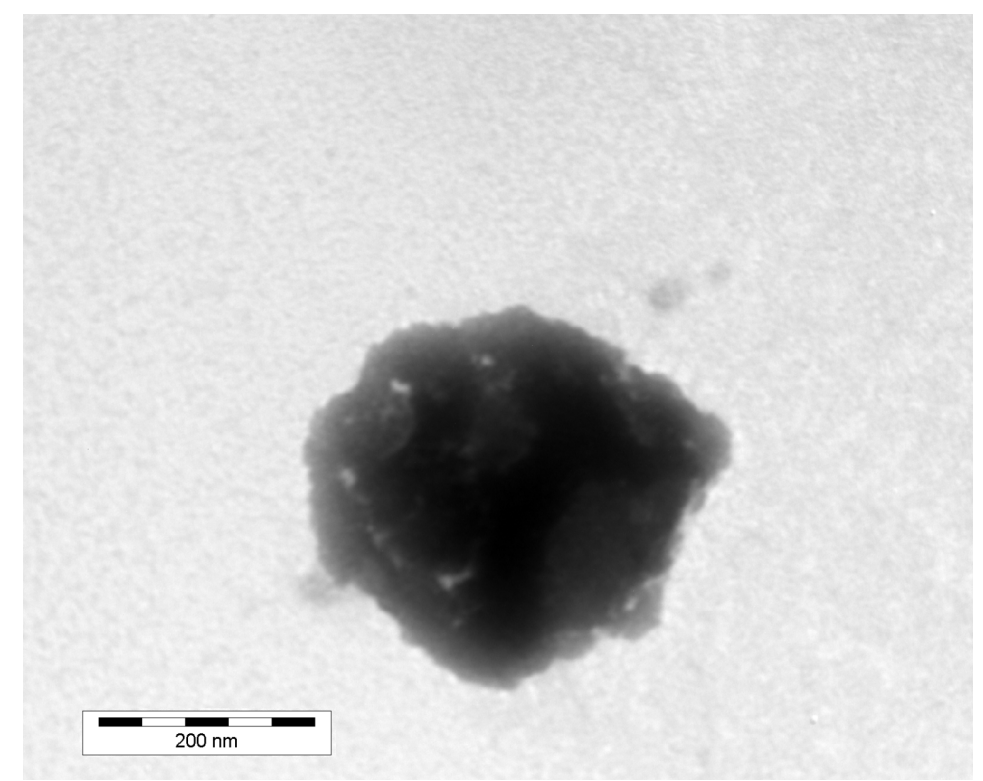

Figure 4. Transmission electron micrograph of BH-loaded CS-MMT/TPP NPs, bar represent $1 \mu \mathrm{m}$.

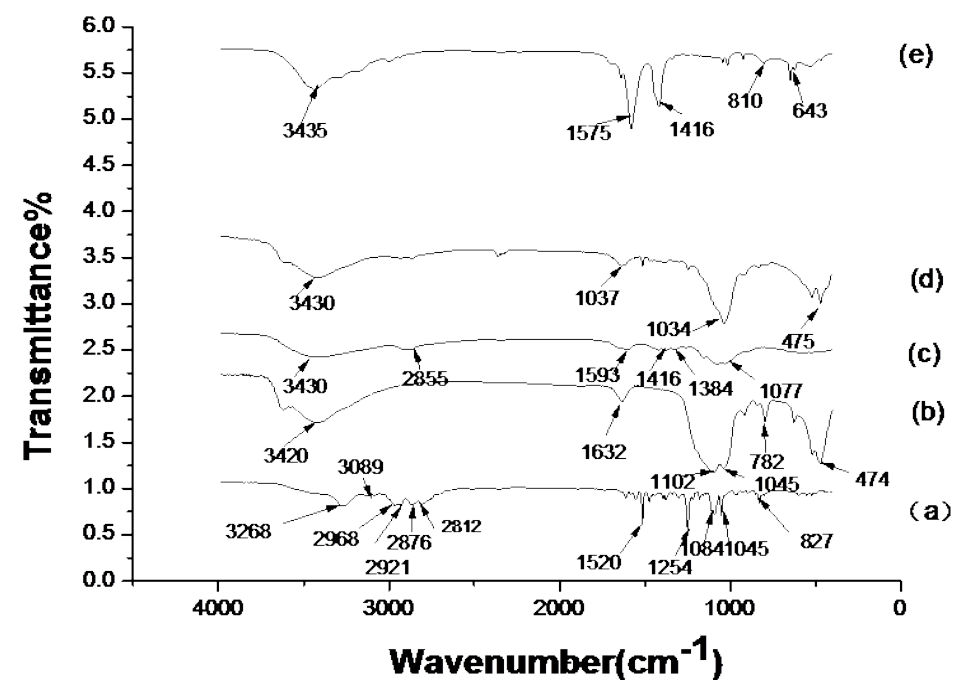

Figure 5. FTIR spectrum of (a) BH, (b) MMT, (c) CS, (d) Physical mixtures of BH, MMT and CS, (e) BH-loaded CS-MMT/TPP NPs. 
spectrum of BH showed several obvious characteristic peaks: the strong peak at $3248 \mathrm{~cm}^{-1}$ belongs to the $-\mathrm{OH}$ stretching vibrations; aromatic $\mathrm{C}=\mathrm{C}$ stretching vibrations are at $1610 \mathrm{~cm}^{-1}$ and $1520 \mathrm{~cm}^{-1}$; aromatic C-H stretching vibrations are at $3089 \mathrm{~cm}^{-1}$; the peaks at $2968 \mathrm{~cm}^{-1}$ and $2961 \mathrm{~cm}^{-1}$ correspond to $-\mathrm{CH}_{3}$ stretching; the peaks at $2876 \mathrm{~cm}^{-1}$ and $2812 \mathrm{~cm}^{-1}$ correspond to $-\mathrm{CH}_{2}$ stretching; C-O-C stretching vibrations are at $1084 \mathrm{~cm}^{-1}$. In MMT spectrum the strong peak at $3420 \mathrm{~cm}^{-1}$ corresponds to the $\mathrm{O}-\mathrm{H}$ stretching vibrations; $\mathrm{Si}-\mathrm{O}$ stretching is at $1102 \mathrm{~cm}^{-1}$ and $1042 \mathrm{~cm}^{-1}$; the intense peaks of $519 \mathrm{~cm}^{-1}$ and $479 \mathrm{~cm}^{-1}$ belong to Fe-O-Si stretching and MgO-Si stretching, respectively; the stretching of Mg-Al-OH is at $782 \mathrm{~cm}^{-1}$; The IR spectrum of chitosan was characterized by the intense peak at $3435 \mathrm{~cm}^{-1}$ corresponded to the combined peak of O-H stretching and intermolecular hydrogen bonding. Specific peaks of chitosan were observed at $1630 \mathrm{~cm}^{-1}$ (amide I) and $1593 \mathrm{~cm}^{-1}$ (amine II). The symmetric stretch of C-O-C is found at around $1077 \mathrm{~cm}^{-1}$, and the intense peak at $1416 \mathrm{~cm}^{-1}$ belongs to C-N stretching.

The spectrum patterns of physical mixtures corresponded simply to the superposition of the IR spectra of betaxolol, montmorillonite and chitosan. The characteristic peaks for BH were almost completely obscured by the very intense and broad peaks of chitosan and montmorillonite. The FT-IR spectrum of BH-loaded CS-MMT/ TPP NPs is different from that of physical mixtures and each pure component. In NPs the tip of peak at 3420 $\mathrm{cm}^{-1}$ was shifted to $3435 \mathrm{~cm}^{-1}$, indicating that hydrogen bonding is enhanced. The $1953 \mathrm{~cm}^{-1}$ peak of N-H blending vibration disappeared and new peaks at $1575 \mathrm{~cm}^{-1}$ and $1416 \mathrm{~cm}^{-1}$ were observed. This spectra changed may be attributed to the electrostatic interaction between chitosan and TPP. In addition, the hydrogen-bond interaction between chitosan and montmorillonite could explain this fact as well.

\subsection{Thermogravimetric Analysis (TG)}

The TG curves of pure components, their corresponding physical mixture, BH-loaded CS/TPP NPs and BHloaded CS-MMT/TPP NPs were showed in Figure 6. There are two distinct changes in the TG profiles of montmorillonite. The first weight loss from $42^{\circ} \mathrm{C}$ to $184^{\circ} \mathrm{C}$ may be due to the free water evaporation. The second weight loss from $480^{\circ} \mathrm{C}$ to $625^{\circ} \mathrm{C}$ is attributed to the structural dehydration. In the curves of simple physical mixture, the weight loss from $228^{\circ} \mathrm{C}$ to $347^{\circ} \mathrm{C}$ corresponds to the decomposition of $\mathrm{BH}$. Other weight losses are the same as those of MMT and CS. The TG patterns of MMT-BH/CS NPs are shown in Figure 6(d)). The first weight loss from $42^{\circ} \mathrm{C}$ to $184^{\circ} \mathrm{C}$ may be attributed to the free water evaporation. The second weight loss from $228^{\circ} \mathrm{C}$ to $347^{\circ} \mathrm{C}$ may be owed to free drug decomposition. The third weight loss from $450^{\circ} \mathrm{C}$ to $520^{\circ} \mathrm{C}$ is attributed to the drug decomposition which was encapsulated into nanoparticles. We can see that the weight loss at around $300^{\circ} \mathrm{C}$ in Figure 6(e) shifts to $500^{\circ} \mathrm{C}$ in Figure 6(d), which may be explained by the fact that the BH is partly encapsulated into the nanoparticles. The last weight loss from $840^{\circ} \mathrm{C}$ to $900^{\circ} \mathrm{C}$ may be due to the destruction of ionic bond between CS and TPP.

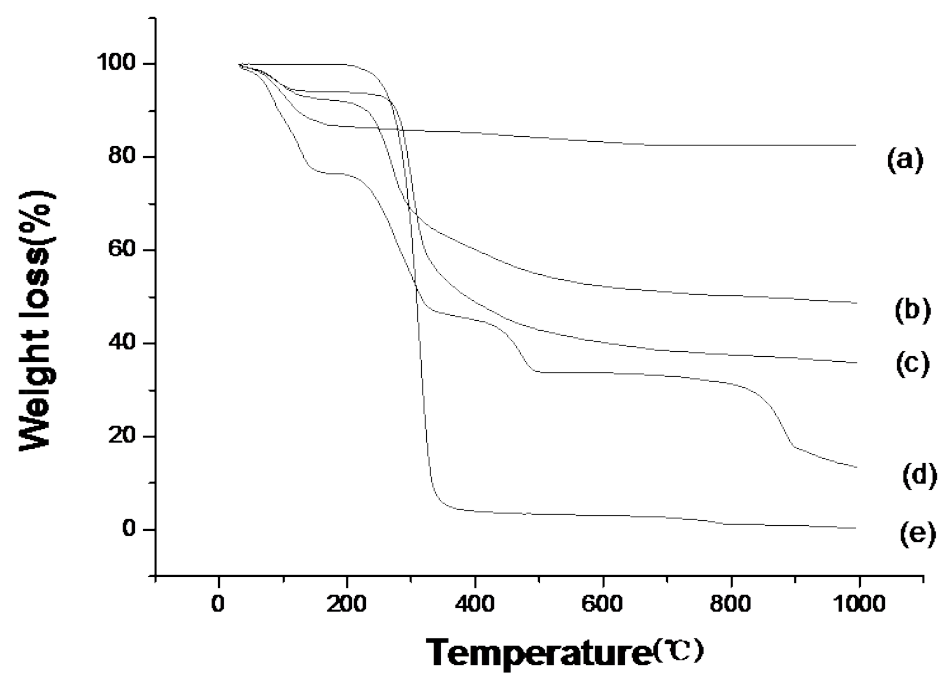

Figure 6. TGA curves of (a) MMT, (b) physical mixture of BH, MMT, CS, (c) CS, (d) BH loading CS-MMT/TPP NPs, (e) BH. 


\subsection{X-Ray Diffratometry}

Figure 7 shows XRD patterns of BH, acid-MMT, CS, their physical mixture, blank nanoparticles and optimized nanoparticles. BH has a specific sharp peak. MMT and CS show the topical reflection at 2-theta $=5.82$ and 20.18, respectively. No characteristic peaks of CS and MMT were found in diffracograms of blank nanoparticles and optimized nanoparticles, which indicated that chitosan nanoparticles were comprised of a dense network structure of interpenetrating polymer chain crosslinked to each other by the electrostatic reaction between TPP and chitosan. The XRD demonstrated the greater disarray in chain alignment in the nanoparticles after cross-link. Therefore, the BH-loaded CS-MMT/TPP NPs were successfully formed.

\subsection{Mucoadhesion Studies}

Mucoadhesion studies of the optimized nanoparticles showed that the nanoparticles revealed the reduction in zeta potential values for CS nanoparticles occurring on mixing with mucin (Figure 8). The reduction may be attributed to the ionic reaction between negatively charged sialic acid residues in mucin and positively charged

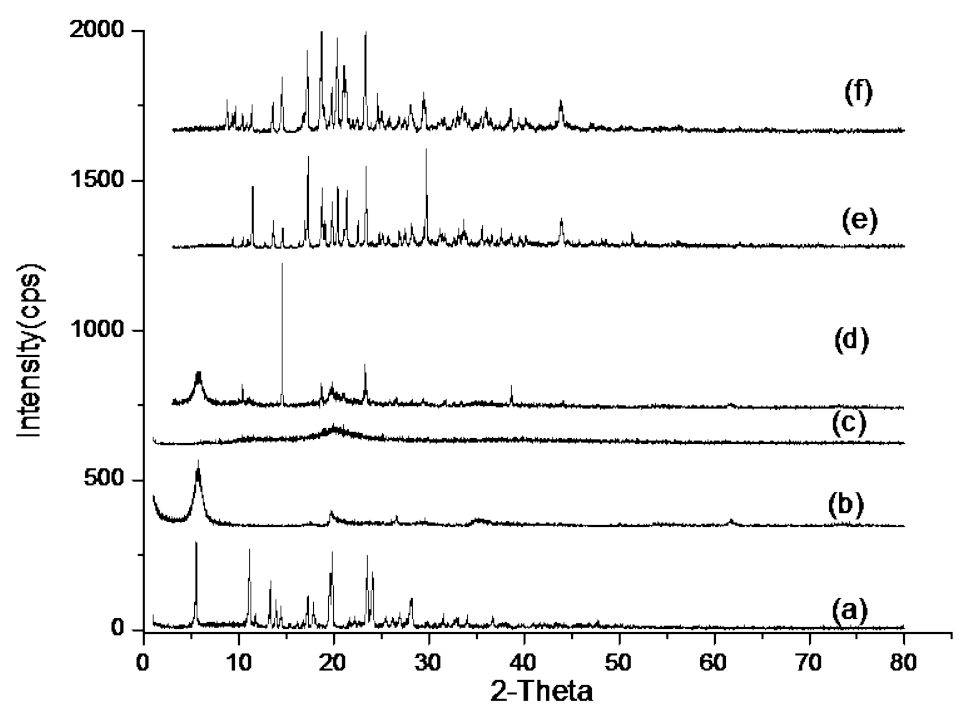

Figure 7. X-ray diffraction of (a) BH; (b) acid-MMT; (c) CS; (d) their physical mixture; (e) blank NPs and (f) oputimized NPs.

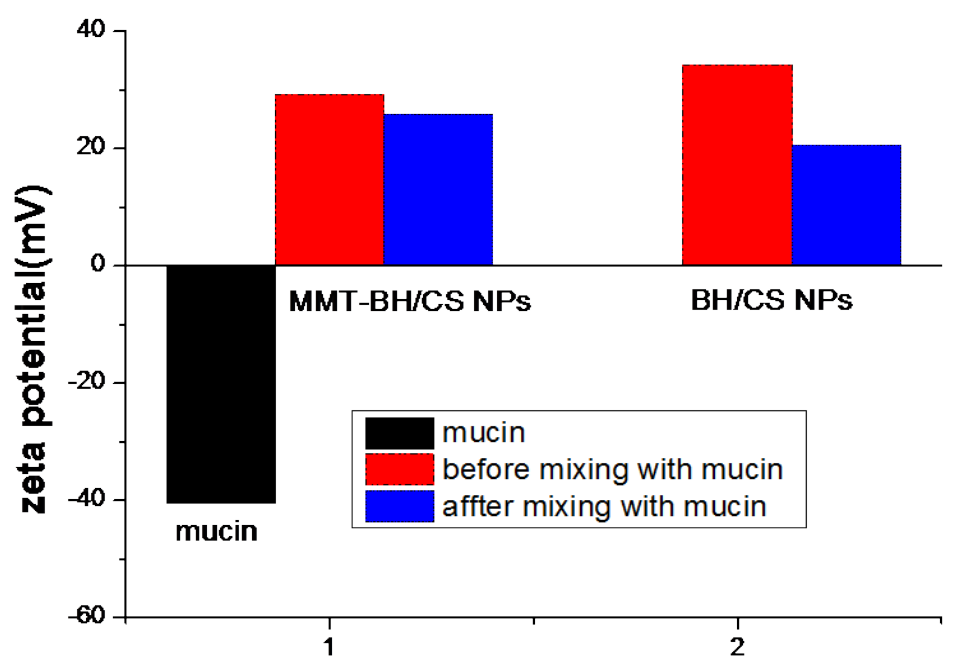

Figure 8. Zeta potential values obtained for mucin and BH/CS and MMT$\mathrm{BH} / \mathrm{CS}$ nanoparticles before and after incubation with mucin. 
amino groups in CS nanoparticles that results in the mucoadhesive properties of the tested nanoparticles. BH/CS nanoparticles showed more reduction in zeta potential values indicating more mucoadhesive compared to $\mathrm{BH}-$ loaded CS-MMT/TPP NPs. This may be attributed to the fact that BH-loaded CS/TPP NPs had higher zeta potential values $(+34 \mathrm{mV})$ than MMT-BH/CS nanoparticles $(+29.18 \mathrm{mV})$.

\subsection{Ocular Irritation Studies}

Figure 9 presents the histopathology of the tested rabbit's eyeballs with various preparations to investigate their influence on cell structure and tissue integrity. The in vivo study showed no sign of irritation or damaging effects in cornea, conunctiva or iris. The scores for conjunctiva congestion of MMT-CS/TPP nanoparticle formulation and control group were grade zero. The cornea and iris scores were zero for all tested samples at all observations (data not shown). Pathology of rabbit eyeballs and lids confirmed the presence of normal ocular surface structure in both optimized nanoparticles cornea and conjunctival epithelial cell maintained normal morphology and integrated epithelium. The basal columnar cell of cornea can be recognized as single lager cell, and is normally packed by tight junction complex (Figure 9(a) \& Figure 9(b)). Similarly, normal levels of scattered polymorphonuclear cells were observed in the conjuctival stroma (Figure 9(c) \& Figure 9(d)), which indicated that there were no signs of inflammation and tissue edema. The treatment of corneas with optimized formulations was exemplified in Figure 9(a), and the histopathology confirmed that no ocular irritating effects were induced by MMT-CS/TPP nanoparticle formulation compared with control group. All tested samples showed a good corneal biocompatibility which demonstrated a preferable ocular tolerance.

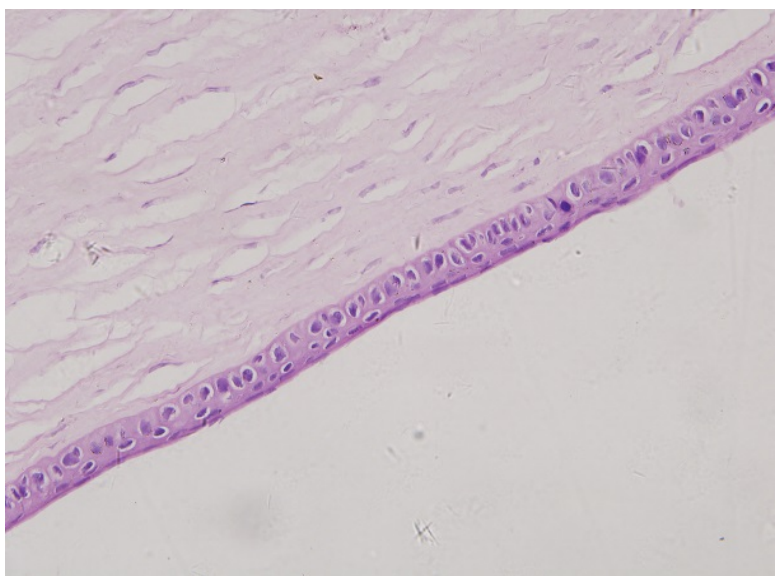

(a)

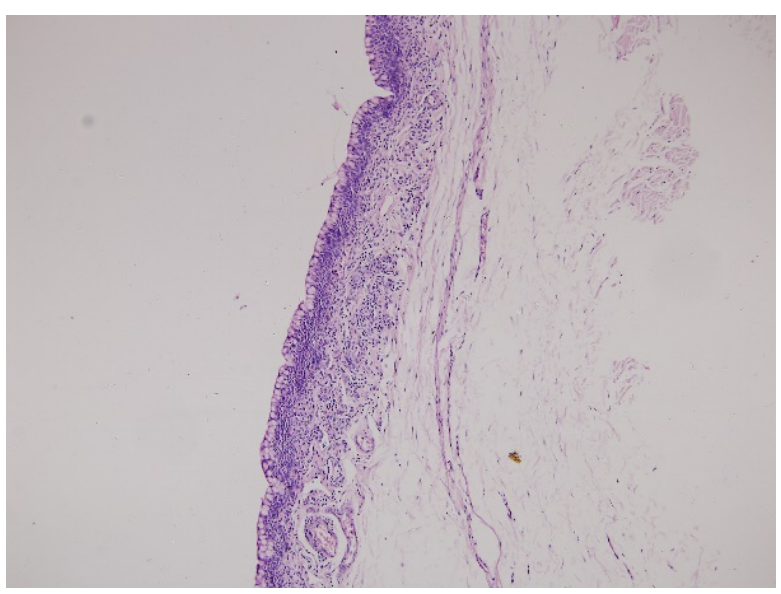

(c)

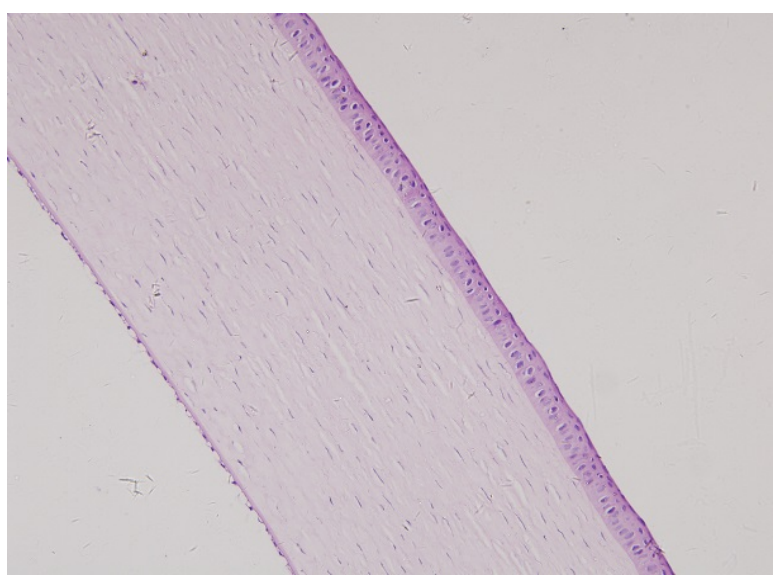

(b)

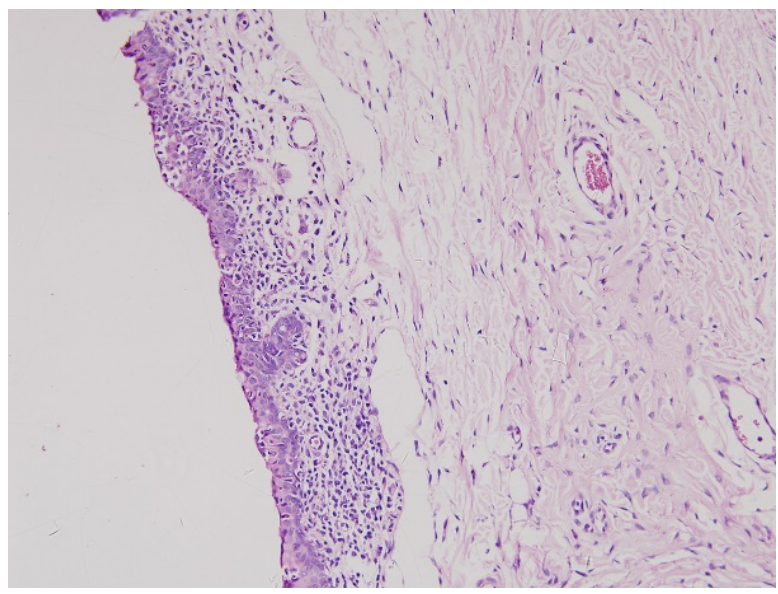

(d)

Figure 9. Histopathology microscopy of the ocular tissues including cornea (a) and (b) and conjunctiva (c) and (d) after treated with different solutions for 7 days. (a) and (c) MMT-BH/CS NPs; (b) and (d) Normal saline. 


\section{Conclusion}

In the present study, the BH loading CS-MMT/TPP nanoparticles were prepared successfully by ionic reaction between CS and TPP based on the positively charged amino groups in CS and the negatively charged phosphate groups in TPP. In addition, the hydroxide radical on the surface of montmorillonite interacting with chitosan through hydrogen bond also played an important role during the preparation of nanoparticles. The drug-loaded nanoparticles consisting of MMT, CS and TPP, showed the enhancement of precorneal retention, sustained release, and high drug-loading capacity. The ratio between TPP and CS was critical for the control of particle size and encapsulation efficiency of nanoparticles. No ocular damage in cornea, conjunctiva or iris was observed. Consequently, these nanocarriers represented a promising approach for the circumvention of the present limitation in ocular drug delivery.

\section{Acknowledgements}

The authors would thank to the youth Natural Science Foundation for financial support (NO.51102052), and thank the eye institute of Shandong province in China for the provision of human immortalized cornea epithelial cell line (HCEC).

\section{References}

[1] Quigley, H.A. and Broman, A.T. (2006) The Number of People with Glaucoma Worldwide in 2010 and 2020. British Journal of Ophthalmology, 90, 262-267. http://www.ncbi.nlm.nih.gov/pmc/articles/PMC1856963/ http://dx.doi.org/10.1136/bjo.2005.081224

[2] Mahalaxmi, D., Samarth, M.M., Shiravadekar, H.S. and Sanghavi, N.M. (1996) Stability-Indicating HPLC Method for Betaxolol HCl and Its Pharmaceutical Dosage Forms. Drug Development and Industrial Pharmacy, 22, 1037-1039. http://www.tandfonline.com/doi/abs/10.3109/03639049609065938\#abstract http://dx.doi.org/10.3109/03639049609065938

[3] Kaur, I.P. and Kanwar, M. (2002) Ocular Preparations: The Formulation Approach. Drug Development and Industrial Pharmacy, 28, 473-493. http://www.tandfonline.com/doi/full/10.1081/DDC-120003445\#abstract http://dx.doi.org/10.1081/DDC-120003445

[4] Hämäläinen, K.M., Kananen, K., Auriola, S., Kontturi, K. and Urtti, A. (1997) Characterization of Paracellular and Aqueous Penetration Routes in Cornea, Conjunctiva and Sclera. Investigative Ophthalmology and Visual Science, 38, 627-634. http://www.ncbi.nlm.nih.gov/pubmed/9071216

[5] Keister, J.C., Cooper, E.R., Missel, P.J., Lang, J.C. and Hager, D.F. (1991) Limits on Optimizing Ocular Drug Delivery. Journal of Pharmaceutical Sciences, 80, 50-53. http://www.ncbi.nlm.nih.gov/pubmed/?term http://dx.doi.org/10.1002/jps.2600800113

[6] Calvo, C.P., Vila-Jato, J.L. and Alonso, M.J. (1997) Novel Hydrophilic Chitosan-Polyethylene Oxide Nanoparticles as Protein Carriers. Journal of Applied Polymer Science, 63, 125-132. http://onlinelibrary.wiley.com/doi/10.1002/\%28SICI\%291097-4628\%2819970103\%2963:1\%3C125::AID-APP13\%3E 3.0.CO;2-4/abstract http://dx.doi.org/10.1002/(SICI)1097-4628(19970103)63:1<125::AID-APP13>3.0.CO;2-4

[7] Kaur, I.P. and Smitha, R. (2002) Penetration Enhancers and Ocular Bioadhesives: Two New Avenues for Ophthalmic Drug Delivery. Drug Development and Industrial Pharmacy, 28, 353-369. http://informahealthcare.com/doi/abs/10.1081/DDC-120002997\%20 http://dx.doi.org/10.1081/DDC-120002997

[8] Ludwig, A. (2005) The Use of Mucoadhesive Polymers in Ocular Drug Delivery. Advances Drug Delivery Reviews, 57, 1595-1639. http://www.ncbi.nlm.nih.gov/pubmed/16198021 http://dx.doi.org/10.1016/j.addr.2005.07.005

[9] Mucha, M. (1997) Rheological Characteristics of Semi-Dilute Chitosan Solutions. Macromolecular Chemistry and Physics, 198, 471-484. http://onlinelibrary.wiley.com/doi/10.1002/macp.1997.021980220/abstract http://dx.doi.org/10.1002/macp.1997.021980220

[10] Wang, W. and Xu, D. (1994) Viscosity and Flow Properties of Concentrated Solutions of Chitosan with Different Degrees of Deacetylation. International Journal of Biological Macromolecule, 16, 149-152.

http://www.ncbi.nlm.nih.gov/pubmed/?term http://dx.doi.org/10.1016/0141-8130(94)90042-6

[11] Alonso, M.J. and Sanchez, A. (2003) The Potential of Chitosan in Ocular Drug Delivery. Journal of Pharmacy and 
Pharmacology, 55, 1451-1463.

http://onlinelibrary.wiley.com/doi/10.1211/0022357022476/abstract;jsessionid=8714D64E9E7D3894BF48FBA2B0D0 912C.f01t04 http://dx.doi.org/10.1211/0022357022476

[12] Bhatta, R.S., Chandasana, H., Chhonker, Y.S., Rathi, C., Kumar, D., Mitra, K. and Shukla, P.K. (2012) Mucoadhesive Nanoparticles for Prolonged Ocular Delivery of Natamycin: In Vitro and Pharmacokinetics Studies. International Journal of Pharmaceutics, 432, 105-112. http://www.sciencedirect.com/science/article/pii/S0378517312004061 http://dx.doi.org/10.1016/j.ijpharm.2012.04.060

[13] Diebold, A. and Calonge, M. (2010) Applications of Nanoparticles in Ophthalmology. Progress in Retinal and Eye Research, 29, 596-609.

http://www.sciencedirect.com/science?_ob=ArticleListURL\&_method=list\&_ArticleListID=-782671355\&_sort=r\&_st $=13 \&$ view $=c \& m d 5=e 5 b f c d 6370882023 e 9 f c 1 c 79 a 37 a e 974 \&$ searchtype $=a$ http://dx.doi.org/10.1016/j.preteyeres.2010.08.002

[14] Motwani, S.K., Chopra, S., Talegaonkar, S., Kohli, K., Ahmad, F.J. and Khar, R.K. (2008) Chitosan-Sodium Alginate Nanoparticles as Submicroscopic Reservoirs for Ocular Delivery: Formulation, Optimization and in Vitro Characterization. European Journal of Pharmaceutics and Biopharmaceutics, 68, 513-525. http://linkinghub.elsevier.com/retrieve/pii/S0939-6411(07)00308-6

[15] Angela, M., Campos, D., Sanchez, A. and Alonso, M.J. (2001) Chitosan Nanoparticles: A New Vehicle for the Improvement of the Delivery of Drugs to the Ocular Surface. Application to Cyclosporin A. International Journal of Pharmaceutics, 224, 159-168. http://linkinghub.elsevier.com/retrieve/pii/S0378517301007608 http://dx.doi.org/10.1016/S0378-5173(01)00760-8

[16] Ennajih, H., Bouhfid, R., Essassi, E.L.M., Bousmina, M. and El Kadib, A. (2012) Chitosan-Montmorillonite Bio-Based Aerogel Hybrid Microspheres. Microporous and Mesoporous Materials, 152, 208-213. http://dx.doi.org/10.1002/chem.201104006

[17] Lin, F.H., Lee, Y.H., Jian, C.H., Wong, J.M., Schiech, M.J. and Wang, C.Y. (2002) A Study of Purified Montmorillonite Intercalated with 5-Fluorouracil as Drug Carrier. Biomaterials, 23, 1981-198.

http://www.ncbi.nlm.nih.gov/pubmed/11996039 http://dx.doi.org/10.1016/s0142-9612(01)00325-8

[18] Zheng, J.P., Luan, L., Wang, H.Y., Xi, L.F. and Yao, K.D. (2007) Study on Ibuprofen/Montmorillonite Intercalation Composites as Drug Release System. Applied Clay Science, 36, 297-301. http://www.sciencedirect.com/science/article/pii/S0169131707000026 http://dx.doi.org/10.1016/j.clay.2007.01.012

[19] Bhatta, R.S., Chandasana, H., Chhonker, Y.S., Rathi, C., Kumar, D., Mitra, K. and Shukla, P.K. (2012) Mucoadhesive Nanoparticles for Prolonged Ocular Delivery of Natamycin: In Vitro and Pharmacokinetics Studies. International Journal of Pharmaceutics, 432, 105-112. http://www.sciencedirect.com/science/article/pii/S0378517312004061 http://dx.doi.org/10.1016/j.ijpharm.2012.04.060

[20] Qi, L.F., Xu, Z.R., Jiang, X., Hu, C.H. and Zou, X.F. (2004) Preparation and Antibacterial Activity of Chitosan Nanoparticles. Carbohydrate Research, 339, 2693-2700. http://www.ncbi.nlm.nih.gov/pubmed/15519328 http://dx.doi.org/10.1016/j.carres.2004.09.007

[21] Mahmoud, A.A., El-Feky, G.S., Kamel, R. and Awad, G.E.A. (2011) Chitosan/Sulfobutylether- $\beta$-Cyclodextrin Nanoparticles as a Potential Approach for Ocular Drug Delivery. International Journal of Pharmaceutics, 413, $229-236$. http://linkinghub.elsevier.com/retrieve/pii/S0378-5173(11)00358-9 http://dx.doi.org/10.1016/j.ijpharm.2011.04.031

[22] Wilhelmus, K.R. (2001) The Draize Eye Test. Survey of Ophthalmology, 45, 493-515. http://www.ncbi.nlm.nih.gov/pubmed/11425356 http://dx.doi.org/10.1016/s0039-6257(01)00211-9

[23] Vandenberg, G.W., Drolet, C., Scott, S.L. and Noue, J.D. (2001) Factors Affecting Protein Release from AlginateChitosan Coacervate Microcapsules during Production and Gastric/Intestinal Simulation. Journal of Controlled Release, 77, 297-307. http://www.sciencedirect.com/science/article/pii/S016836590100517X http://dx.doi.org/10.1016/S0168-3659(01)00517-X

[24] Quan, G. and Tao, W. (2007) Chitosan Nanoparticle as Protein Delivery Carrier—Systematic Examination of Fabrication Conditions for Efficient Loading and Release. Colloid and Surfaces B: Biointerfaces, 59, 24-34. http://www.sciencedirect.com/science/article/pii/S0927776507001671

[25] Kawashima, Y., Yamamoto, H., Takeuchi, H. and Kuno, Y. (2000) Mucoadhesive DL-Lactide/Glycolide Copolymer Nanoparticles Coated with Chitosan to Improve Oral Delivery of Elcatonin. Pharmaceutical Development and Technology, 5, 77-85. http://informahealthcare.com/doi/abs/10.1081/PDT-100100522\%20

http://dx.doi.org/10.1081/PDT-100100522 
[26] Oyarzun-Ampuero, F.A., Brea, J., Loza, M.I., Torres, D. and Alonso, M.J. (2009) Chitosan-Hyaluronic Acid Nanoparticles Loaded with Heparin for the Treatment of Asthma. International Journal of Pharmaceutics, 2, 122-129. http://www.sciencedirect.com/science/article/pii/S0378517309002129 http://dx.doi.org/10.1016/j.ijpharm.2009.04.009

[27] Schiffelers, R.M., Woodle, M.C. and Scaria, P. (2004) Pharmaceutical Prospects for RNA Interference. Pharmacy Research, 21, 1-7. http://link.springer.com/article/10.1023\%2FB\%3APHAM.0000012145.49054.6c 